\title{
Untangling Coefficients for Lorentz Violation
}

\author{
Kenneth A. Amandolia ${ }^{1}$, Charles D. Lane ${ }^{1 *}$
}

The Lorentz-violating Standard-Model Extension is a general framework with coefficients that describe potential violations of special and general relativity. Many constraints that have been placed on the Standard-Model Extension (SME) involve combinations of multiple coefficients. We untangle some of these constraints to obtain simpler bounds on the basic SME coefficients that appear in the fundamental action of the framework and focus on the electron $c_{\mu v}$ coefficients, which affect the electron's dispersion relation in a Lorentz-violating vacuum. We find several constraints are some of the most sensitive yet reported.

\section{INTRODUCTION}

A physical theory is Lorentz symmetric if it predicts that all inertial reference frames are physically equivalent. That is, the theory predicts that results of an experiment performed in one inertial frame will be identical to results performed in another inertial frame that has different orientation or velocity. This is a key principle behind the theory of special relativity, and hence lies at the root of all modern physical theories. However, it is possible that this principle could be broken in a theory that operates at a more fundamental scale (Kostelecky \& Samuel, 1989). Over the last several decades there have been a tremendous number of experiments performed to test the validity of Lorentz symmetry (Kostelecky \& Russell, Data Tables for Lorentz and CPT Violation, 2011). All experiments to date indicate that Lorentz symmetry is perfect; bounds on different potential violations range from parts per $10^{2}$ to parts per $10^{33}$. Nevertheless, due to its foundational role in modern physics and potential sensitivity to physics beyond the standard model, it is important to test Lorentz symmetry with as much precision as possible.

For the sake of comparing different experimental signals, it is useful to have a general theoretical framework that allows for Lorentz symmetry to be violated. The SME (Colladay \& Kostelecky, 1997; Colladay \& Kostelecky, 1998; Kostelecky, 2004) is an effective quantum field theory that preserves observer Lorentz symmetry while allowing general violation of particle Lorentz symmetry (Tasson, 2016). The types of Lorentz violation that could arise are parametrized in the SME action by sets of coefficients such as $a_{\mu}$,

${ }^{1}$ Physics, Astronomy, and Geology Department, Berry College, 2277 Martha Berry Hwy NW, Mount Berry, GA 30149

*To whom correspondence should be addressed: clane@berry.edu

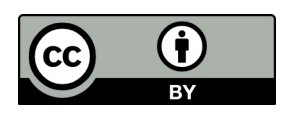

Except where otherwise noted, this work is licensed under https://creativecommons.org/licenses/by/4.0

doi:10.22186/jyi.34.5.26-30 $b_{\mu}, c_{\mu \nu}, \ldots$ for each particle species. These may be thought of as vectors and tensors that are nonzero even in a vacuum. An experiment that is, in some sense, aligned with one of these may give different results from an experiment that is not aligned, and therefore the existence of these vectors and tensors violates Lorentz symmetry.

We focus in this work on the $c_{\mu v}$ tensor for electrons, which perturbs the electron's energy-momentum dispersion relation away from its conventional expression. The perturbed dispersion relation implies, in turn, that an electron's kinetic energy will depend on its direction of travel and speed; for example, in a suitable limit, the kinetic energy of a free electron with velocity $\vec{v}$ is given by $K=\frac{1}{2} m\left(v^{2}+\vec{v} \cdot c \cdot \vec{v}\right)$, where $c$ represents the matrix of $c_{j k}$. This orientation dependence constitutes a violation of Lorentz symmetry. Detailed formulas for these perturbations are cumbersome and beyond the scope of this paper, but may be found in the literature (Colladay \& Kostelecky, 2001).

Some experimental signals involve only a single SME coefficient; however, most involve linear combinations of multiple coefficients. When there are enough linearly independent combinations of coefficients that have been bounded, we may extract bounds on the individual coefficients that appear. In this work, we derive a method for extracting such bounds and apply it to the $c_{\mu \nu}$ coefficients that are associated with electrons.

\section{METHODS}

We use two methods to untangle bounds on SME coefficients. The first is mostly graphical while the second uses standard statistical results and some index notation. Each method has its own strengths and weaknesses. The first is transparent for simple cases but difficult to visualize in higher dimensions. In some situations, it may not yield the strongest applicable bounds. The second is less transparent but easily extends to higher dimensions. Moreover, slight variations may be used for different sets of statistical assumptions.

\section{Method 1: Graphical}

The first method we use is best described with a simple example. An experiment (Hohensee et al., 2013) using dysprosium spectroscopy yielded the following pair of bounds involving electronassociated coefficients $c_{X Y}$ and $c_{Y Z}$ : 


$$
\begin{aligned}
& 0.94 c_{X Y}-0.35 c_{Y Z}=(-0.4 \pm 2.8) \times 10^{-17} \\
& 0.35 c_{X Y}+0.94 c_{Y Z}=(3.2 \pm 7.0) \times 10^{-17}
\end{aligned}
$$

Note that each of these is consistent with zero, indicating that these are constraints on Lorentz violation rather than observation of it. It is convenient to introduce shorter names for the relevant parts of these coefficients, $a:=10^{17} c_{X Y}$ and $b:=10^{17} c_{Y Z}$, in terms of which the experimental bounds are

$$
\begin{aligned}
& 0.94 a-0.35 b=-0.4 \pm 2.8, \\
& 0.35 a+0.94 b=3.2 \pm 7.0 .
\end{aligned}
$$

Our goal is to determine the bounds on $a$ and $b$ that are implied by this pair of equations, and hence obtain clean bounds on the original coefficients $c_{X Y}$ and $c_{Y Z}$.

Equations (2) may be expressed graphically as in Figure 1. The grey parallelogram represents the values allowed by Eq. (2) with borders given by the uncertainties. The dashed lines represent the best measurement results, i.e., the results with uncertainties set to zero. (Note that the $a$ and $b$ axes are not drawn with the same scale.)

Figure 2 shows the same graph but with different labels to highlight different aspects. The small heavy square and its projections onto the $a$ and $b$ axes represent the best values of those variables, $\langle a\rangle$ and $\langle b\rangle$. The heavy circles and their projections represent the uncertainty range of each variable, $a=\langle a\rangle \pm \partial a$ and $b=<b> \pm \partial b$.

We can use linear algebra to calculate each of these values numerically. First, write the bounds in the form

$$
M\left(\begin{array}{l}
a \\
b
\end{array}\right)=\left(\begin{array}{c}
-0.4 \pm 2.8 \\
3.2 \pm 7.0
\end{array}\right),
$$

where matrix $M=\left(\begin{array}{cc}0.94 & -0.35 \\ 0.35 & 0.94\end{array}\right)$. There are two " \pm " signs in this expression, indicating that it represents 4 similar expressions, each of which defines one of the corners of the parallelogram in Figure 2. For example, point A corresponds to choosing a "+" sign in each place. Then the parallelogram corners are found with the matrix inverse:

$$
\left(\begin{array}{l}
a \\
b
\end{array}\right)=M^{-1}\left(\begin{array}{c}
-0.4 \pm 2.8 \\
3.2 \pm 7.0
\end{array}\right)=\left(\begin{array}{cc}
0.94 & 0.35 \\
-0.35 & 0.94
\end{array}\right)\left(\begin{array}{c}
-0.4 \pm 2.8 \\
3.2 \pm 7.0
\end{array}\right) .
$$

Corner A corresponds to the choice of both "+" signs while corner C corresponds to the choice of both "." signs:

$$
\begin{aligned}
& \left(\begin{array}{l}
a_{A} \\
b_{A}
\end{array}\right)=M^{-1}\left(\begin{array}{c}
-0.4+2.8 \\
3.2+7.0
\end{array}\right)=\left(\begin{array}{l}
5.8 \\
8.7
\end{array}\right), \\
& \left(\begin{array}{l}
a_{C} \\
b_{C}
\end{array}\right)=M^{-1}\left(\begin{array}{c}
-0.4-2.8 \\
3.2-7.0
\end{array}\right)=\left(\begin{array}{l}
-4.3 \\
-2.4
\end{array}\right) .
\end{aligned}
$$

Similarly, corners B and D correspond to choices of signs that are not the same:

$$
\begin{aligned}
& \left(\begin{array}{l}
a_{B} \\
b_{B}
\end{array}\right)=M^{-1}\left(\begin{array}{c}
-0.4-2.8 \\
3.2+7.0
\end{array}\right)=\left(\begin{array}{c}
0.6 \\
10.6
\end{array}\right), \\
& \left(\begin{array}{l}
a_{D} \\
b_{D}
\end{array}\right)=M^{-1}\left(\begin{array}{c}
-0.4+2.8 \\
3.2-7.0
\end{array}\right)=\left(\begin{array}{c}
0.9 \\
-4.4
\end{array}\right) .
\end{aligned}
$$

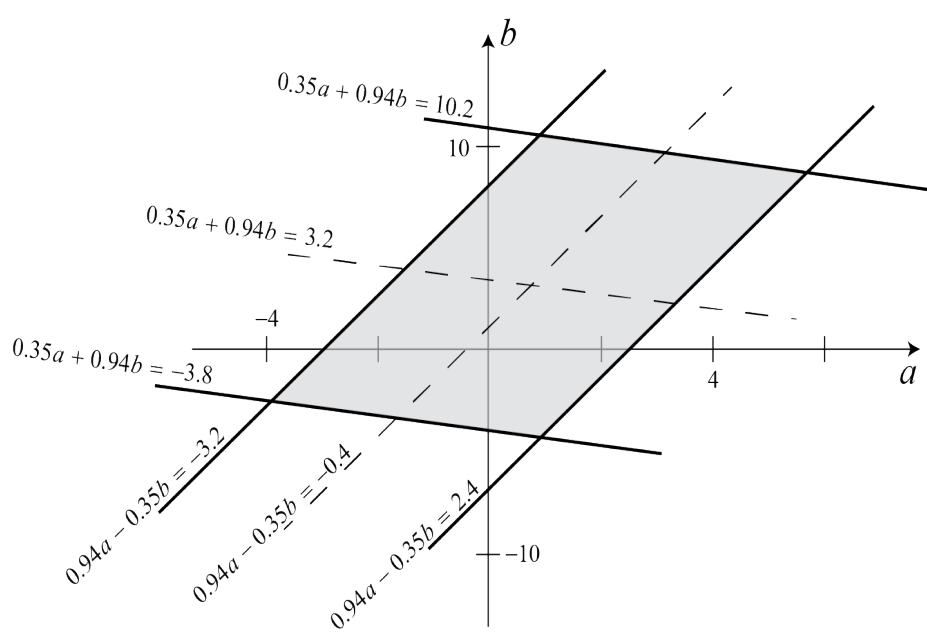

Figure 1. Current bounds on linear combinations of coefficients $a$ and $\boldsymbol{b}$. The grey parallelogram represents the values allowed by Eq. (2) with borders given by the uncertainties. The dashed lines represent the best measurement results, i.e., the results with uncertainties set to zero. (Note that the $a$ and $b$ axes are not drawn with the same scale.)

Finally, the best values $\langle a\rangle$ and $\langle b\rangle$, can be found by evaluating Eq. (4) but with uncertainties set to zero:

$$
\left(\begin{array}{c}
<a> \\
\langle b\rangle
\end{array}\right)=M^{-1}\left(\begin{array}{c}
-0.4 \\
3.2
\end{array}\right)=\left(\begin{array}{l}
0.7 \\
3.1
\end{array}\right) \text {. }
$$

The bound on $a$ is determined by corners A and C only; corners B and $\mathrm{D}$ are irrelevant, as can be seen from the diagram. Similarly, the bound on $b$ is determined by corners B and D only. Combining Eqs. (5), (6), and (7) then gives $a=0.7 \pm 5.0$ and $b=3.1 \pm 7.5$, from which we can immediately write down the untangled bounds on the original SME coefficients:

$$
\begin{aligned}
& c_{X Y}=(0.7 \pm 5.0) \times 10^{-17}, \\
& c_{Y Z}=(3.1 \pm 7.5) \times 10^{-17} .
\end{aligned}
$$

This procedure may be extended to a greater number, $n \geq 3$, of tangled bounds involving a more SME coefficients, provided the number of linearly independent bounds is the same as the number of coefficients that appear. Unfortunately, it soon becomes difficult to visualize --- the analogous diagram to Fig. 1 involves an $n$-dimensional parallelotope (Coxeter, 1973). Nevertheless, the schemes of finding corners using matrix inversion may be applied.

For example, an experiment (Pruttivarasin, et al., 2015) with trapped calcium ions yielded a set of four bounds on four linearly independent combinations of four SME electron-associated coefficients:

$$
\left(\begin{array}{cccc}
-0.16 & 0.33 & -0.92 & -0.16 \\
-0.04 & -0.32 & -0.35 & 0.88 \\
0.29 & -0.38 & -0.73 & -0.48 \\
-0.31 & -0.65 & 0.07 & -0.69
\end{array}\right)\left(\begin{array}{c}
c_{X-Y} \\
c_{X Y} \\
c_{X Z} \\
c_{Y Z}
\end{array}\right)=\left(\begin{array}{c}
1 \pm 10 \\
2.4 \pm 7.4 \\
5.9 \pm 9.5 \\
7 \pm 12
\end{array}\right) \times 10^{-19} .
$$

These bounds define a 4-dimensional parallelotope with 16 verti- 
ces. The center of the parallelotope corresponds to the best values $\left\langle c_{X-Y}\right\rangle$, etc., of the SME coefficients, and can be calculated by inverting the matrix above and setting the uncertainties each to be zero. Each of the 16 vertices corresponds to a set of choices for the four " \pm " signs that appear. The most difficult part of this analysis is determining which vertices are most relevant for which SME coefficients. Alternately, we may assume that each vertex is relevant in turn, and then select the largest constraints among the vertices for each coefficient. In either case, the method is straightforward if tedious.

\section{Method 2: Formulas with Index Notation}

As noted above, the graphical method is unwieldy whenever there are more than two SME coefficients involved. For these situations, we can use formulas from standard statistical analysis to determine constraints (Taylor, 1996).

Furthermore, Method 1 yields uncertainty values that may or may not be appropriate for a given situation. They are the uncertainties that would appear if the original measurements were correlated in such a way that the uncertainty in one measurement always enhances the uncertainties in other measurements. We remind the reader that uncertainties do not represent absolute limits; rather, they represent the range of likely measurement results. These ideas can be seen working together, for example, in Figure 2, the grey parallelogram represents the range of likely measurements, and the corners $\mathrm{A}$ and $\mathrm{C}$ that we used to estimate uncertainty in $a$ are at the extremes of the parallelogram. Measurements of $a$ are more likely to lie in a somewhat smaller region unless the original measurements have a special correlation. The results of Method 1, therefore, represent a sort of maximum possible uncertainty that could pertain. These are appropriate as conservative estimates if correlations between measurements are not well known.

However, there may be situations in which we can assume that the individual tangled bounds are statistically independent from each other. In such cases, tighter uncertainties are appropriate, and we should use the error-propagation method of "adding in quadrature" (Taylor, 1996).

Calculations reflecting these issues are best described with general variables for the tangled and untangled bounds. Let $\left\{q_{i}\right\}$ denote a set of $n$ SME coefficients that appear in a set of bounds. Our goal is to write each of these in the form $q_{j}=<q_{j}> \pm \partial q_{j}$, where $\left\langle q_{j}>\right.$ is the best measurement value of $q_{j}$ and $\partial q_{j}$ is the uncertainty in $q_{j}$. Meanwhile, each experimental result is written in the form $r_{j}=\left\langle r_{j}\right\rangle \pm \partial r_{j}$ where $\left\{r_{j}\right\}$ denote the linear combinations of the $q_{j^{\prime}}$ This means that there is a matrix $M$ such that each $r_{j}=$ $\sum_{k} M_{j k} q_{k}$. Combining all this notation, we may write the set of measured bounds in the form

$$
\sum_{k} M_{j k} q_{k}=r_{j}=<r_{j}> \pm \delta r_{j}
$$

for $j=1, \ldots, n$. We can invert matrix $M$ if there are $n$ linearly independent bounds. This yields the expressions

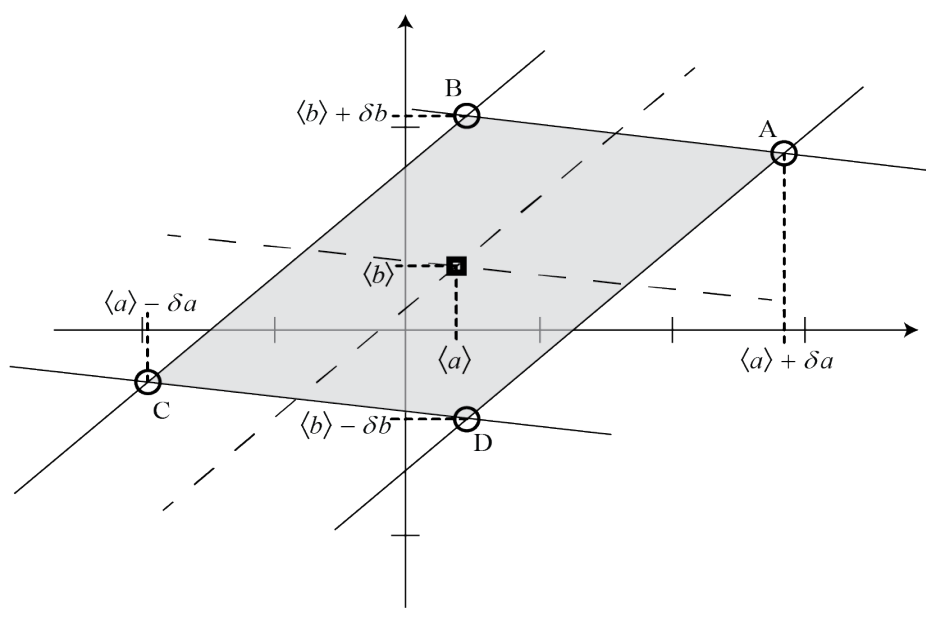

Figure 2. The same graph as Figure 1 but with different labels to highlight different aspects. The small heavy square and its projections onto the $a$ and $b$ axes represent the best values of those variables, $<a>$ and $<b$ $>$. The heavy circles and their projections represent the uncertainty range of each variable, $a=\langle a\rangle \pm \partial a$ and $b=\langle b\rangle \pm \partial b$.

$$
<q_{k}>=\sum_{\ell}\left(M^{-1}\right)_{k \ell}<r_{\ell}>
$$

for the best measurement values. The Method 1 process also yields the formulas

$$
\left(\delta q_{k}\right)_{\max }=\sum_{\ell}\left|\frac{\partial q_{k}}{\partial r_{\ell}} \delta r_{\ell}\right|=\sum_{\ell}\left|\left(M^{-1}\right)_{k \ell} \delta r_{\ell}\right|
$$

for maximal possible uncertainties. As an example, consider again the set of bounds from dysprosium spectroscopy discussed first in Method 1. There are $n=2$ SME coefficients: $\left(\begin{array}{l}q_{1} \\ q_{2}\end{array}\right)=\left(\begin{array}{l}c_{X Y} \\ c_{Y Z}\end{array}\right)$.

The linear combinations of these that are directly measured are

$$
\left(\begin{array}{l}
r_{1} \\
r_{2}
\end{array}\right)=\left(\begin{array}{l}
0.94 q_{1}-0.35 q_{2} \\
0.35 q_{1}+0.94 q_{2}
\end{array}\right) \text {, corresponding to matrix } M=\left(\begin{array}{cc}
0.94 & -0.35 \\
0.35 & 0.94
\end{array}\right) \text {. }
$$

The bounds themselves are $\left(\begin{array}{l}<r_{1}> \pm \delta r_{1} \\ <r_{2}> \pm \delta r_{2}\end{array}\right)=\left(\begin{array}{c}(-0.4 \pm 2.8) \times 10^{-17} \\ (3.2 \pm 7.0) \times 10^{-17}\end{array}\right)$ Using

the matrix inverse $M^{-1}=\left(\begin{array}{cc}0.94 & 0.35 \\ -0.35 & 0.94\end{array}\right)$ we get the best values

$\left(\begin{array}{l}\left\langle c_{X Y}\right\rangle \\ \left\langle c_{Y Z}\right\rangle\end{array}\right)=\left(\begin{array}{l}\left\langle q_{1}\right\rangle \\ \left\langle q_{2}\right\rangle\end{array}\right)=\left(\begin{array}{l}0.7 \times 10^{-17} \\ 3.1 \times 10^{-17}\end{array}\right)$ and maximum uncertainties

$\left(\begin{array}{l}\delta c_{X Y} \\ \delta c_{Y Z}\end{array}\right)_{\max }=\left(\begin{array}{l}\delta q_{1} \\ \delta q_{2}\end{array}\right)_{\max }=\left(\begin{array}{l}5.0 \times 10^{-17} \\ 7.5 \times 10^{-17}\end{array}\right)$.

In the opposite situation, we may know that the experimental measures of $r_{j}$ are uncorrelated with each other. In that case, we may apply the "adding in quadrature" formula for propagation of statistically independent random uncertainty (Taylor, 1996),

$$
\left(\delta q_{k}\right)_{\text {quad }}=\sqrt{\sum_{\ell}\left[\frac{\partial q_{k}}{\partial r_{\ell}} \delta r_{\ell}\right]^{2}}=\sqrt{\sum_{\ell}\left[\left(M^{-1}\right)_{k \ell} \delta r_{\ell}\right]^{2}} .
$$




\begin{tabular}{|c|c|c|c|c|c|}
\hline $\begin{array}{l}\text { Standard-Model } \\
\text { Extension Tilde } \\
\text { Coefficient }\end{array}$ & $\begin{array}{c}\text { Standard-Model } \\
\text { Extension Coef- } \\
\text { ficient }\end{array}$ & $\begin{array}{l}\text { Result with Max } \\
\text { Uncertainty }\end{array}$ & $\begin{array}{l}\text { Result with Quadra- } \\
\text { ture Uncertainty }\end{array}$ & System & Reference \\
\hline$\sim c_{-} / m$ & $c_{X-Y}$ & $(-2 \pm 43) \times 10^{-19}$ & $(-2 \pm 23) \times 10^{-19}$ & Trapped Ca ions & (Pruttivarasin et al., 2015) \\
\hline$\sim c_{Z} /(2 m)$ & $c_{X Y}$ & $(-8 \pm 23) \times 10^{-19}$ & $(-8 \pm 12) \times 10^{-19}$ & Trapped $\mathrm{Ca}$ ions & (Pruttivarasin et al., 2015) \\
\hline$\sim c_{Y} /(2 m)$ & $c_{X Z}$ & $(-3 \pm 13) \times 10^{-19}$ & $(-3 \pm 8) \times 10^{-19}$ & Trapped $\mathrm{Ca}$ ions & (Pruttivarasin et al., 2015) \\
\hline$\sim c_{X} /(2 m)$ & $c_{Y Z}$ & $(-2 \pm 12) \times 10^{-19}$ & $(-2 \pm 7) \times 10^{-19}$ & Trapped $\mathrm{Ca}$ ions & (Pruttivarasin et al., 2015) \\
\hline$\sim c_{-} / m$ & $c_{X-Y}$ & $(3 \pm 7) \times 10^{-17}$ & $(3 \pm 6) \times 10^{-17}$ & $\begin{array}{l}\text { Dysprosium } \\
\text { spectroscopy }\end{array}$ & (Hohensee et al., 2013) \\
\hline$\sim c_{Y} /(2 m)$ & $c_{X Z}$ & $(9 \pm 12) \times 10^{-17}$ & $(9 \pm 11) \times 10^{-17}$ & $\begin{array}{l}\text { Dysprosium } \\
\text { spectroscopy }\end{array}$ & (Hohensee et al., 2013) \\
\hline$\sim c_{z} /(2 m)$ & $c_{X Y}$ & $(0.7 \pm 5) \times 10^{-17}$ & $(0.7 \pm 4) \times 10^{-17}$ & $\begin{array}{l}\text { Dysprosium } \\
\text { spectroscopy }\end{array}$ & (Hohensee et al., 2013) \\
\hline$\sim c_{X} /(2 m)$ & $c_{Y Z}$ & $(3 \pm 8) \times 10^{-17}$ & $(3 \pm 7) \times 10^{-17}$ & $\begin{array}{l}\text { Dysprosium } \\
\text { spectroscopy }\end{array}$ & (Hohensee et al., 2013) \\
\hline$\sim c_{T X} /(2 m)$ & $c_{T X}$ & $(6 \pm 11) \times 10^{-15}$ & $(6 \pm 8) \times 10^{-15}$ & $\begin{array}{l}\text { Dysprosium } \\
\text { spectroscopy }\end{array}$ & (Hohensee et al., 2013) \\
\hline$\sim c_{T Y} /(2 m)$ & $c_{T Y}$ & $(-8 \pm 8) \times 10^{-13}$ & $(-8 \pm 8) \times 10^{-13}$ & $\begin{array}{l}\text { Dysprosium } \\
\text { spectroscopy }\end{array}$ & (Hohensee et al., 2013) \\
\hline$\sim c_{T Z} /(2 m)$ & $c_{T Z}$ & $(19 \pm 18) \times 10^{-13}$ & $(19 \pm 17) \times 10^{-13}$ & $\begin{array}{l}\text { Dysprosium } \\
\text { spectroscopy }\end{array}$ & (Hohensee et al., 2013) \\
\hline
\end{tabular}

Table 1. Untangled bounds to electron-associated $c$ coefficients. The tilde coefficients are linear combinations of the base coefficients, and are defined in Table P41 of the Data Tables (Kolselecky \& Russell, 2011).

In the special case of the example above, this gives slightly tighter uncertainties

$$
\left(\begin{array}{l}
\delta c_{X Y} \\
\delta c_{Y Z}
\end{array}\right)_{\text {quad }}=\left(\begin{array}{l}
\delta q_{1} \\
\delta q_{2}
\end{array}\right)_{\text {quad }}=\left(\begin{array}{l}
3.6 \times 10^{-17} \\
6.6 \times 10^{-17}
\end{array}\right) .
$$

\section{RESULTS AND DISCUSSION}

These methods may be applied to the four clusters of tangled bounds that appear in Table D6 of the Data Tables for Lorentz violation (Kostelecky \& Russell, 2011). One cluster comes from study of trapped calcium ions (Pruttivarasin et al., 2015) and the other three come from an experiment involving dysprosium spectroscopy (Hohensee et al., 2013).

All the resulting untangled bounds are listed in Table 1. They are grouped according to the cluster of bounds from which they originate. In addition to the base SME coefficients, we express each bound in terms of the tilde coefficients (Bluhm, Kostelecky, Lane, \& Russell, 2003; Kostelecky \& Lane, 1999) summarized in Table P41 of the Data Tables.

Several coefficients are bounded multiple times in Table 1. In Table 2, we extract the tightest bound on each tilde coefficient that arises from this work. Two of the bounds, on $\sim c_{-}$and $\sim c_{z}$, are the tightest known from any experiment, and therefore may replace the values currently listed in Table S2 of the Data Tables.

\section{ACKNOWLEDGEMENTS}

This work was supported in part by Berry College.

\begin{tabular}{|c|c|c|c|}
\hline $\begin{array}{c}\text { Standard- } \\
\text { Model Exten- } \\
\text { sion Tilde } \\
\text { Coefficient }\end{array}$ & $\begin{array}{l}\text { Maximum } \\
\text { Sensitivity }\end{array}$ & System & Reference \\
\hline$\sim c_{-}$ & $2 \times 10^{-21} \mathrm{GeV}$ & $\begin{array}{c}\text { Trapped Ca } \\
\text { ions }\end{array}$ & $\begin{array}{c}\text { (Pruttivarasin, et } \\
\text { al., 2015) }\end{array}$ \\
\hline$\sim c_{X}$ & $1 \times 10^{-21} \mathrm{GeV}$ & $\begin{array}{l}\text { Trapped Ca } \\
\text { ions }\end{array}$ & $\begin{array}{c}\text { (Pruttivarasin, et } \\
\text { al., 2015) }\end{array}$ \\
\hline$\sim c_{Y}$ & $1 \times 10^{-21} \mathrm{GeV}$ & $\begin{array}{l}\text { Trapped } \mathrm{Ca} \\
\text { ions }\end{array}$ & $\begin{array}{c}\text { (Pruttivarasin, et } \\
\text { al., 2015) }\end{array}$ \\
\hline$\sim c_{Z}$ & $2 \times 10^{-21} \mathrm{GeV}$ & $\begin{array}{c}\text { Trapped } \mathrm{Ca} \\
\text { ions }\end{array}$ & $\begin{array}{c}\text { (Pruttivarasin, et } \\
\text { al., 2015) }\end{array}$ \\
\hline$\sim c_{T X}$ & $1 \times 10^{-17} \mathrm{GeV}$ & $\begin{array}{l}\text { Dy spectros- } \\
\text { copy }\end{array}$ & $\begin{array}{c}\text { (Hohensee, et al., } \\
\text { 2013) }\end{array}$ \\
\hline$\sim c_{T Y}$ & $0.8 \times 10^{-15} \mathrm{GeV}$ & $\begin{array}{l}\text { Dy spectros- } \\
\text { copy }\end{array}$ & $\begin{array}{c}\text { (Hohensee, et al., } \\
\text { 2013) }\end{array}$ \\
\hline$\sim c_{T Z}$ & $2 \times 10^{-15} \mathrm{GeV}$ & $\begin{array}{l}\text { Dy spectros- } \\
\text { copy }\end{array}$ & $\begin{array}{c}\text { (Hohensee, et al., } \\
\text { 2013) }\end{array}$ \\
\hline
\end{tabular}

Table 2. Maximal sensitivities to tilde combinations of SME coefficients that result from this analysis. The entries in the first column are the usual tilde-c combinations. Note that some of these sensitivities will replace values in Table S2 of the Data Tables (Kostelecky and Russell, 2011). 


\section{REFERENCES}

Bluhm, R., Kostelecky, V. A., Lane, C. D., \& Russell, N. (2003). Probing Lorentz and CPT Violation with Space-Based Experiments. Phys. Rev. D, 68, 125008. doi:10.1103/PhysRevD.68.125008

Colladay, D., \& Kostelecky, V. A. (1997). CPT Violation and the Standard Model. Phys. Rev. D, 55, 6760-6774. doi:10.1103/PhysRevD.55.6760

Colladay, D., \& Kostelecky, V. A. (1998). Lorentz-Violating Extension of the Standard Model. Phys. Rev. D, 58, 116002. doi:10.1103/PhysRevD.58.116002

Colladay, D., \& Kostelecky, V. (2001). Cross-sections and Lorentz violation. Phys. Lett. B, 511, 209-217. doi:10.1016/S0370-2693(01)00649-9

Coxeter, H. (1973). Regular Polytopes (3rd ed.). Dover Publications.

Hohensee, M. A., Leefer, N., Budker, D., Harabati, C., Dzuba, V. A., \& Flambaum, V. V. (2013). Limits on Violations of Lorentz Symmetry and the Einstein Equivalence Principle using Radio-Frequency Spectroscopy of Atomic Dysprosium. Phys. Rev. Lett., 111, 050401. doi:10.1103/PhysRevLett.111.050401

Kostelecky, V. A. (2004). Gravity, Lorentz Violation, and the Standard Model. Phys. Rev. D, 69, 105009. doi:10.1103/PhysRevD.69.105009

Kostelecky, V. A., \& Lane, C. D. (1999). Constraints on Lorentz Violation from Clock-Comparison Experiments. Phys. Rev. D, 60, 116010. doi:10.1103/ PhysRevD.60.116010

Kostelecky, V. A., \& Russell, N. (2011). Data Tables for Lorentz and CPT Violation. Rev. Mod. Phys., 83, 11-31. doi:10.1103/RevModPhys.83.11

Kostelecky, V. A., \& Samuel, S. (1989). Spontaneous Breaking of Lorentz Symmetry in String Theory. Phys. Rev. D, 39, 683. doi:10.1103/PhysRevD.39.683

Pruttivarasin, T., Ramm, M., Porsev, S. G., Tupitsyn, I. I., Safronova, M. Hohensee, M. A., Haeffner, H. (2015). A Michelson-Morley Test of Lorentz Symmetry for Electrons. Nature, 517, 592. doi:10.1038/nature14091

Tasson, J. D. (2016). The Standard-Model Extension and Gravitational Tests. Symmetry, 8, 111.

Taylor, J. R. (1996). An Introduction to Error Analysis: The Study of Uncertainties in Physical Measurements (2nd ed.). University Science Books. 